\title{
Descent Rate Models of the Synchronization of the Quasi-Biennial Oscillation by the Annual Cycle in Tropical Upwelling
}

\author{
KyLASH RAJENDRAN AND IRENE M. MOROZ \\ Mathematical Institute, University of Oxford, Oxford, United Kingdom \\ SCOTT M. OSPREY AND PETER L. READ \\ Atmospheric, Oceanic and Planetary Physics, University of Oxford, Oxford, United Kingdom
}

(Manuscript received 9 September 2017, in final form 5 February 2018)

\begin{abstract}
The response of the quasi-biennial oscillation (QBO) to an imposed mean upwelling with a periodic modulation is studied, by modeling the dynamics of the zero wind line at the equator using a class of equations known as descent rate models. These are simple mathematical models that capture the essence of QBO synchronization by focusing on the dynamics of the height of the zero wind line. A heuristic descent rate model for the zero wind line is described and is shown to capture many of the synchronization features seen in previous studies of the QBO. It is then demonstrated using a simple transformation that the standard HoltonLindzen model of the QBO can itself be put into the form of a descent rate model if a quadratic velocity profile is assumed below the zero wind line. The resulting nonautonomous ordinary differential equation captures much of the synchronization behavior observed in the full Holton-Lindzen partial differential equation. The new class of models provides a novel framework within which to understand synchronization of the QBO, and we demonstrate a close relationship between these models and the circle map well known in the mathematics literature. Finally, we analyze reanalysis datasets to validate some of the predictions of our descent rate models and find statistically significant evidence for synchronization of the QBO that is consistent with model behavior.
\end{abstract}

\section{Introduction}

The quasi-biennial oscillation $(\mathrm{QBO})$ is the dominant mode of variability of zonal-mean zonal winds in the tropical stratosphere between 100 and $5 \mathrm{hPa}$. It consists of alternating bands of descending westerly and easterly wind regimes that form in the tropical upper stratosphere and descend to the tropical tropopause at an average rate of approximately $1 \mathrm{~km} \mathrm{month}^{-1}$. A review of the basic properties and dynamics of the $\mathrm{QBO}$ is given in Baldwin et al. (2001).

The period of the QBO has long been thought to be somehow related to the annual cycle (Dunkerton and Delisi 1985; Dunkerton 1990). The onset months of QBO westerlies and easterlies have been observed to display a seasonal preference. Pascoe et al. (2005), for example, found in their analysis of ERA-40 data that both westerly and easterly QBO onsets at $44 \mathrm{hPa}$

Corresponding author: K. Rajendran, rajendran@maths.ox.ac.uk tended to occur preferentially during Northern Hemisphere summer. Hampson and Haynes (2004) used a 3D mechanistic model to study the phase evolution of the QBO and found evidence for phase alignment of the QBO resulting from the influence of the annual cycle in tropical upwelling. Krismer et al. (2013) studied the seasonal evolution of the QBO in a full general circulation model and found that the descent of the QBO was affected by a combination of seasonal upwelling, seasonal variations in wave forcing, and the influence of the stratopause semiannual oscillation.

It has been suggested that the seasonal tendency of QBO onsets may be due to an interaction with the annual cycle in tropical upwelling caused by the wavedriven Brewer-Dobson (BD) circulation (Kinnersley and Pawson 1996). Because of the hemispheric asymmetry in topography and land distribution, there is a greater amount of extratropical wave generation during Northern Hemisphere winter as opposed to Southern Hemisphere 
winter. As a result, the strength of the tropical upwelling branch of the BD circulation displays a seasonal cycle (Seviour et al. 2012). Seasonal changes in the strength of upwelling then modulate the effective resistance to the descent of the QBO shear zones, resulting in a seasonal variation in the QBO descent rate.

In a recent study (Rajendran et al. 2016, hereafter R16), the present authors studied the interaction of the QBO with seasonally varying vertical advection caused by an analog of the BD circulation, using the one-dimensional HoltonLindzen (HL) model of the QBO (Holton and Lindzen 1972; Plumb 1977). We found that interactions with the annual cycle resulted in a modulation in the average period of the QBO, indicating that the QBO was synchronized by the BD circulation. Consequently, the QBO also displayed a seasonal tendency in monthly onsets, in line with the findings of previous studies. Intriguingly, we also observed complex variations in the oscillation period of the QBO in the model (see Figs. 3, 4 in R16). These variations displayed a particular structure, wherein the QBO periods were either (i) locked to an integer multiple of the forcing period; (ii) arranged as $n$ cycles, such that the sum of every $n$ periods was an integer multiple of the forcing period; or (iii) continuous, with the average QBO period being an irrational multiple of the forcing period (or at least a rational multiple with a large denominator).

In this paper we attempt to interpret the abovementioned observations by studying a class of equations that we call descent rate models. These models are simple ordinary differential equations that describe the rate of descent of the QBO shear zones in time. [Note: we use the term westerly (easterly) shear zone to denote a contiguous region in the stratosphere throughout which the zonalmean zonal wind blows entirely from the west (east)]. We will show that the dynamics of the QBO period described above are accurately reproduced in these simple models. Furthermore, we will demonstrate that the HL equation itself can, under suitable approximations, be reduced to the form of a descent rate model. Thus, most of the complex behavior of the QBO in the HL equation can be captured by a single ordinary differential equation, achieving a large reduction in complexity with no significant loss in dynamical detail. The descent models are analytically transparent and therefore much easier to interrogate in terms of understanding the mechanisms of synchronization. We will show that the descent rate models are closely linked to the circle map, which is a mathematical model well known from dynamical systems theory. This association provides a solid theoretical foundation to understanding synchronization of the QBO.

The layout of the rest of this paper is as follows. In section 2 we introduce a heuristic model for the descent rate and consider the relevant factors required to model the wave forcings adequately at different heights. We solve the descent rate model for two candidate wave-forcing profiles and find that the behavior of the QBO in the models is very similar to that of the HL equation studied in R16. We demonstrate in section 3 that the HL equation can be put into descent rate form via a simple change of coordinates and a quadratic approximation of the velocity profile. Again, the results of this simple model are found to correspond strongly to the results found in R16. We then discuss the link between the QBO descent rate models and the circle map, and we use the models to illustrate the mechanism behind QBO frequency locking. In section 4 we consider observational evidence for the clustering of QBO periods in reanalysis datasets and show that statistically significant clustering behavior is indeed observable in the real atmosphere. These results dovetail well with the findings from the idealized models. Finally, we discuss the implications of our results in section 5 .

\section{A heuristic descent rate model}

\section{a. Formulation}

Figure 1a depicts sample output from the HL model of the QBO. Easterly winds have been shaded and the zero wind contour has been darkened. The position of the zero wind contour can be traced out by following the point marked $z_{0}^{*}$ in Fig. 1b, which shows zonal wind variations with height at a particular time. The point $z_{0}^{*}$ is known as the zero wind line. (Throughout this paper we use asterisks to denote dimensional quantities.) The idea behind a descent rate equation, then, is to model the temporal rate of change of the zero wind line, $d z_{0}^{*} / d t^{*}$, rather than the velocity profile itself.

We now construct a heuristic model for the dynamics of $z_{0}^{*}$. Consider the case where the QBO is forced by two identical waves, as well as upwelling and diffusion. A shear zone forms in the upper levels of the stratosphere and descends, driven by preferential dissipation of the waves at different heights. The balance of wave forces at the height of $z_{0}^{*}$ is such that the net contribution of wave forcing is always downward; this will be discussed in more detail later. Because the wave driving the descent of the shear zone experiences greater dissipation above the zero wind line than below it, absolute zonal wind velocities just above $z_{0}^{*}$ are always larger than absolute zonal wind velocities just below $z_{0}^{*}$. The resulting curvature of the flow at $z_{0}^{*}$ implies that diffusion exerts a downward force on $z_{0}^{*}$. In contrast, the tendency of upwelling is to impede the descent of $z_{0}^{*}$. Thus, we posit the following model for $z_{0}^{*}$ dynamics:

$$
\frac{d z_{0}^{*}}{d t^{*}}=-D^{*}+\left[w_{c}^{*}+w_{a}^{*} \cos \left(\frac{2 \pi t^{*}}{360}\right)\right]-A_{w}^{*} G\left(z_{0}^{*}\right)
$$


(a)

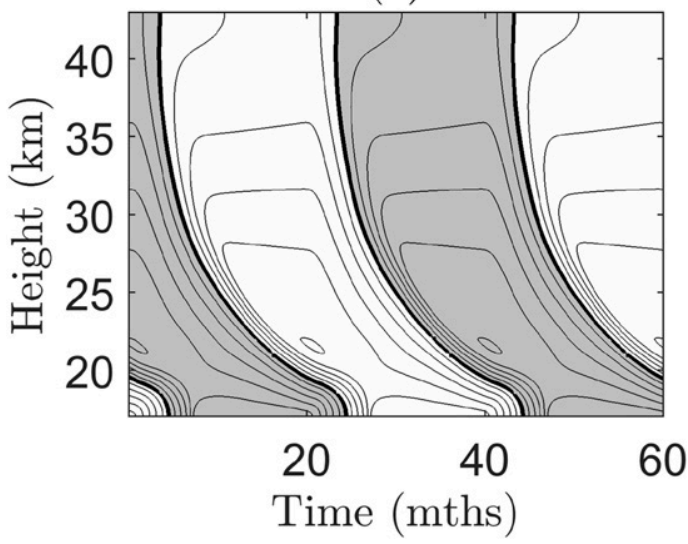

(b)

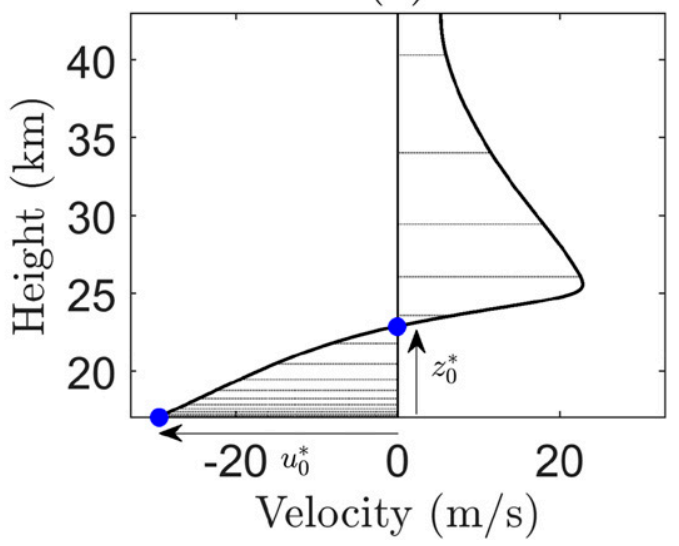

FIG. 1. (a) Contour plot of zonal wind velocities from the HL model. Easterly winds are shaded and the contour interval is $5 \mathrm{~m} \mathrm{~s}^{-1}$. The zero wind line has been darkened. (b) Profile view of zonal wind variation with height at a fixed time. The height of the zero wind line and the tropopause zonal wind velocity have been labeled.

with time $t^{*}$ measured in days. Here we have modeled diffusion resulting from small-scale processes as a constant downward tendency of strength $D^{*}$. Upwelling has been separated into constant and annually varying components $w_{c}^{*}$ and $w_{a}^{*}$, respectively. The length of a year is taken to be 360 days for convenience so that the length of each month can be taken to be 30 days. The wave forcing is expressed as the product of the wave amplitude $A_{w}^{*}\left(\mathrm{~m} \mathrm{~s}^{-1}\right)$ with a wave profile $G\left(z_{0}^{*}\right)$ that is defined below. Since we have chosen the waves to be identical, the wave forcing structure for descending QBO westerlies and QBO easterlies is the same, and so we can use the single evolution equation [(1)] for both cases. The height domain is chosen to be between $z_{b}^{*}=17 \mathrm{~km}$ and $z_{t}^{*}=43 \mathrm{~km}$. For simplicity we assume a periodic domain so that when $z_{0}^{*}$ passes below the tropopause at $z_{b}^{*}=17 \mathrm{~km}$, it maps automatically to the midstratosphere at $z_{t}^{*}=43 \mathrm{~km}$, where it is assumed that new QBO shear zones form. This behavior approximates the destruction of the underlying shear zone as $z_{0}^{*}$ descends to the lower boundary and the immediate formation of a new shear zone aloft. The approximation is reasonable if there is a negligible time difference between the destruction of a shear zone at the lower boundary and its reformation in the midstratosphere as a new shear layer, which is a fair description of the dynamics of the QBO in the HoltonLindzen model.

We nondimensionalize (1) by introducing a height scale $H^{*}=6.5 \mathrm{~km}$ and a time scale $T^{*}=1$ day. We can then define nondimensional time and zero wind height variables via $t^{*}=T^{*} t$ and $\left\{z_{0}^{*}, z_{b}^{*}, z_{t}^{*}\right\}=17 \mathrm{~km}+H^{*}\left\{z_{0}, z_{b}, z_{t}\right\}$ respectively. Substituting into (1) and rearranging gives

$\frac{d z_{0}}{d t}=-D+\left[w_{c}+w_{a} \cos \left(\frac{2 \pi t}{360}\right)\right]-A_{w} G\left(z_{0}\right)$
Here, the dimensionless parameters $D, w_{c}, w_{a}$, and $A_{w}$ are given by

$$
\left\{D, w_{c}, w_{a}, A_{w}\right\}=\frac{t^{*}}{H^{*}}\left\{D^{*}, w_{c}^{*}, w_{a}^{*}, A_{w}^{*}\right\} .
$$

To get a feel for an appropriate form for the wave profile $G_{0}$, we consider the balance of wave forces at the zero wind line as a westerly shear zone descends. This is depicted in Fig. 2, where the horizontal solid red and dashed blue arrows at the zero wind line denote contributions from the easterly and westerly waves, respectively.

When a new westerly shear zone forms in the midstratosphere (Fig. 2a), there is a large easterly shear zone separating the zero wind line from the source of the waves at the tropopause. As such, while both waves are dissipated as they propagate through the shear zone toward $z_{0}$, the easterly wave is absorbed much more than the westerly wave. The easterly wave forcing at $z_{0}$ is therefore much weaker than the westerly wave forcing at $z_{0}$. This results in a large net westerly acceleration, which pushes the zero wind line down.

As the zero wind line descends to lower levels (Fig. 2b), the forcing amplitude of both waves increases, since the zero wind line is now closer to the source region of the waves. As before, there is a discrepancy in wave absorption as a result of the prevailing easterly winds below the zero wind line. However, the magnitude of this difference is now reduced, since the amplitude of the easterly wave gains disproportionately from the reduced extent of the easterly shear zone as compared to the amplitude of the westerly wave. The net result is that the total wave forcing is still westerly, but the magnitude is reduced as compared to the upper-level case discussed earlier. The reduction in strength of the net wave forcing continues all the way down to the tropopause (Fig. 2c), at 
(a)

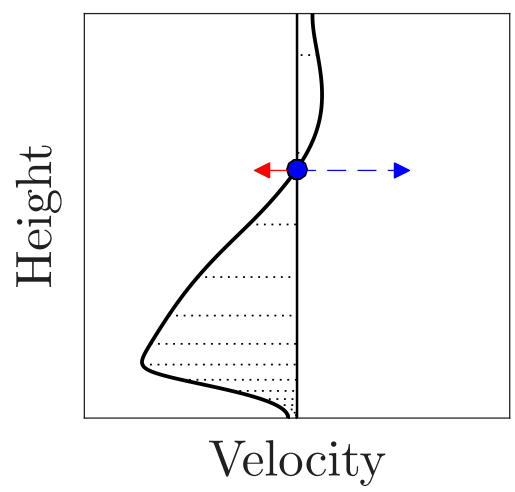

(b)

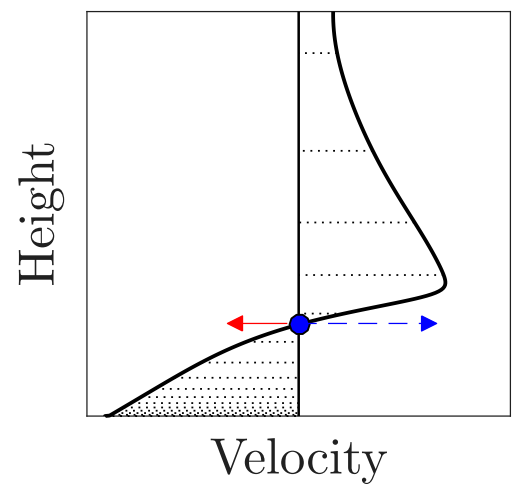

(c)

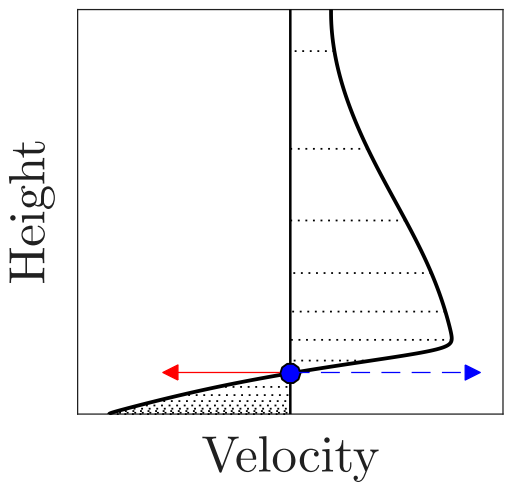

FIG. 2. Force balance of wave driving at the zero wind line for different heights, over the course of the formation and descent of a westerly shear zone. Refer to text for details.

which point the contributions of both waves are almost equal and so the net westerly acceleration is very weak.

While we ignore the effect of density stratification in this study, we note that its inclusion would not change the qualitative picture described above; the amplitude of each wave would simply gain a positive exponential factor as a result of density decreases in height.

Figures $3 \mathrm{a}$ and $3 \mathrm{~b}$ display two candidate profiles for $G\left(z_{0}\right)$ over the simulation domain $z_{0} \in\left[z_{b}, z_{t}\right]=[0,4]$. In the first profile (Fig. 3a), the total wave forcing is close to zero when $z_{0}$ is at the tropopause and increases monotonically with height, eventually becoming almost constant at higher levels. The profile is a sigmoid curve modeled by

$$
G_{0}\left(z_{0}\right)=\exp \left(-b e^{-c z_{0}}\right)
$$

which is a Gompertz function with shape parameters $b=10$ and $c=3$. The profile was chosen to reflect the fact that one wave is essentially trapped in the lower stratosphere, and so in the upper half of the domain there is very little counterbalancing of the acceleration and descent induced by the free wave.

The second candidate wave profile (Fig. 3b) is a sine function given by

$$
G_{0}\left(z_{0}\right)=\sin \left(\frac{\pi z}{4}\right) .
$$

This profile is continuous across the periodic boundary at $z_{0}=0,4$, whereas the Gompertz profile [(4)] is discontinuous. Note that wave forcing at the zero wind line is never small at high levels in reality, because the zero wind line is created only after the upper-level winds experience significant acceleration and switch signs. Hence, in a sense $z_{0}$ is always created "fully formed," with a nonzero initial velocity. This means that a discontinuity in $d z_{0} / d t$ across the periodic boundary is to be expected. Nevertheless, for purposes of comparison it proves useful to consider an example of a $G_{0}$ profile that is continuous across the periodic boundary.

\section{b. Results}

We solve (2) numerically using the forward Euler method, with a time step of $\Delta t=0.2$. The values of $w_{c}=$ 0.004 and $w_{a}=0.002$ are chosen based on physically reasonable values of the corresponding dimensional variables, which are $w_{c}=0.3 \mathrm{~mm} \mathrm{~s}^{-1}$ and $w_{a}=0.15 \mathrm{~mm} \mathrm{~s}^{-1}$, respectively. The quantity $D$ is set equal to $w_{c}+w_{a}=0.006$ in order to counterbalance the upwelling maximum and to prevent the zero wind line from ever ascending. (Ascent past $z_{0}=4$ would undermine the physical meaningfulness of the assumption of circular geometry, and so we remove this possibility from the outset.) The initial condition is set as $z_{0}(0)=0.9$.

Figures $4 \mathrm{a}$ and $4 \mathrm{~b}$ display sample evolutions of $z_{0}$ using the Gompertz and sine wave forcing profiles, respectively, for a wave amplitude set at $A_{w}=0.01$. Blue and red lines in Figs. $4 \mathrm{a}$ and $4 \mathrm{~b}$ represent the descent of westerly and easterly shear zones, respectively.

In both cases the descent of the zero wind lines mimics the descent of the QBO. In the Gompertz case (Fig. 4a), the (a)

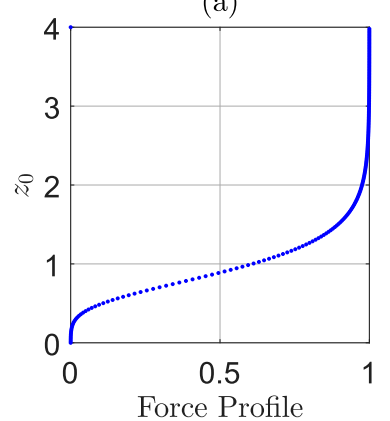

(b)

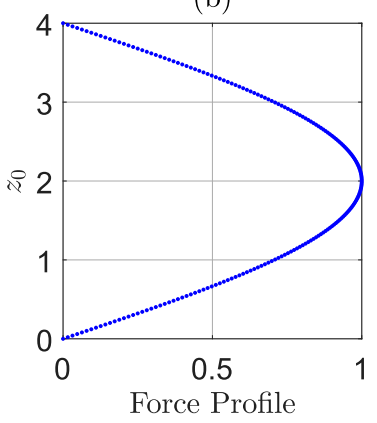

FIG. 3. Candidate profiles of the variation of $G_{0}\left(z_{0}\right)$ : (a) Gompertz and (b) sine profiles. See text for details. 
(a) Gompertz

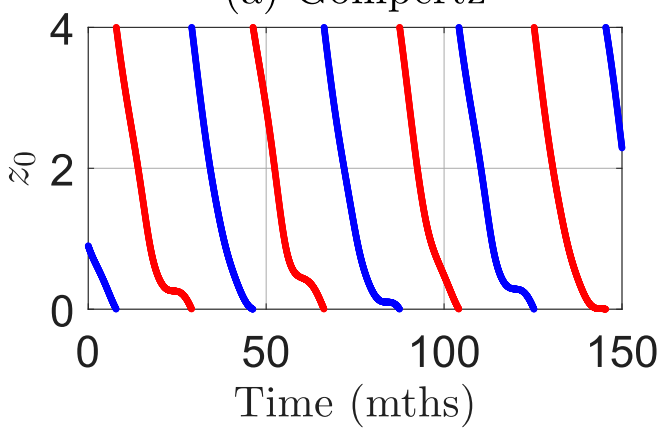

(b) Sine

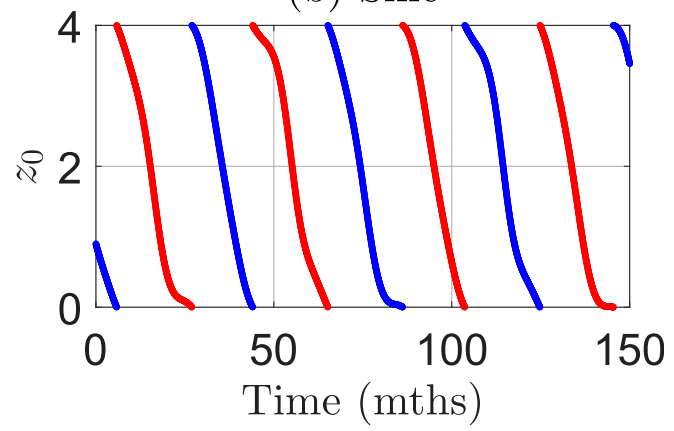

FIG. 4. Sample solutions of the heuristic descent model, using the (a) Gompertz and (b) sine wave profiles. Parameters are set at $A_{w}=0.01, D=0.006, w_{c}=0.004$, and $w_{a}=0.002$. The location of $z_{0}$ over time has been plotted for each profile. Descending westerly (blue line) and easterly (red line) shear zones are shown.

descent of $z_{0}$ is uniform across the upper levels and then slows near the lower boundary as the net wave acceleration reduces. The effects of varying upwelling strength can be seen in the varying descent rates near the bottom boundary, where the wave forcing is weak and upwelling effects are significant.

Similar behavior is seen in the sine case (Fig. 4b), except there is significant variation near the upper boundary as well, because of the reduction of the wave forcing there. The average oscillation periods are quite similar, however, at 38.7 and 39.6 months for the Gompertz and sine cases, respectively.

Having elucidated the basic model behavior, we performed a parameter sweep by varying the value of $A_{w}$ in small increments between $A_{w}=0.003$ and $A_{w}=$ 0.02 . A total of 10000 different values of $A_{w}$ were studied for each of the two wave profiles. Each simulation was run for 50000 model days, including a start-up runtime of 20000 days to allow time for transients to disappear. The corresponding set of QBO periods was then derived by measuring the time between westerly onsets at $z_{0}=0.5$.

Figures 5 and 6 plot the set of QBO periods obtained as described above against the corresponding values of $A_{w}$, in the Gompertz and sine wave forcing cases, respectively. The plots reveal intricate patterns in the detailed QBO period structure and are strongly reminiscent of Figs. 3 and 4 in R16. There are prominent locking regions at multiples of the upwelling period $(24,36,48$, and 60 months for the $A_{w}$ range considered here). Outside of these regions, the QBO is either in a multicycle state-identifiable as the white bands within which the QBO period takes on a small set of finite values-or in a quasi-periodic state wherein the QBO "fills out" a band of periods. The variation of the QBO period within these regions can be quite large. For example, in the Gompertz case (Fig. 5) the range of QBO periods just before the 24-month locking region is 8 months.
These results show that the heuristic descent rate model successfully recreates many of the QBO synchronization phenomena documented in R16.

\section{Descent rate model of the HL equation}

\section{a. Formulation}

We now show how the Holton-Lindzen equation can be transformed into a descent rate equation. Following Holton and Lindzen (1972) and Plumb (1977), the evolution of the zonal-mean zonal wind $\bar{u}^{*}$ is given by

$$
\begin{aligned}
\frac{\partial \bar{u}^{*}}{\partial t^{*}}= & -w^{*} \frac{\partial \bar{u}^{*}}{\partial z^{*}}+\Lambda_{0}^{*} \frac{\partial^{2} \bar{u}^{*}}{\partial z^{* 2}} \\
& +\frac{F * N * \mu_{0}^{*}}{k_{0}^{*}\left(\bar{u}^{*}-c^{*}\right)^{2}} \exp \left[-\int_{z_{b}^{*}}^{z^{*}} \frac{N^{*} \mu_{0}^{*}}{k_{0}^{*}\left(\bar{u}^{*}-c^{*}\right)^{2}} d z^{*}\right] \\
& -\frac{F * N * \mu_{0}^{*}}{k_{0}^{*}\left(\bar{u}^{*}+c^{*}\right)^{2}} \exp \left[-\int_{z_{b}^{*}}^{z^{*}} \frac{N^{*} \mu_{0}^{*}}{k_{0}^{*}\left(\bar{u}^{*}+c^{*}\right)^{2}} d z^{*}\right],
\end{aligned}
$$

where we denote dimensional quantities by asterisks. Here $t^{*}$ represents time in days, $z^{*}$ is height in kilometers, and $w^{*}$ is vertical velocity. The buoyancy frequency $N^{*}$ and diffusion coefficient $\Lambda_{0}^{*}$ are also defined accordingly. We have adopted the formulation of Plumb (1977), wherein the zonal-mean flow is forced by two identical gravity waves of wavenumber $k_{0}^{*}$ traveling in opposite directions with horizontal phase speeds $\pm c^{*}$, respectively. The maximum amplitudes of the wave momentum fluxes are given by $\pm F^{*}$, respectively $\left(\mathrm{m}^{2} \mathrm{~s}^{-2}\right)$, and $\mu_{0}^{*}$ is the dissipation rate of the waves. Appropriate values of $N^{*}, \mu_{0}^{*}, k_{0}^{*}, c^{*}$, and $\Lambda_{0}^{*}$ are listed in Table 1 , together with the values of the 


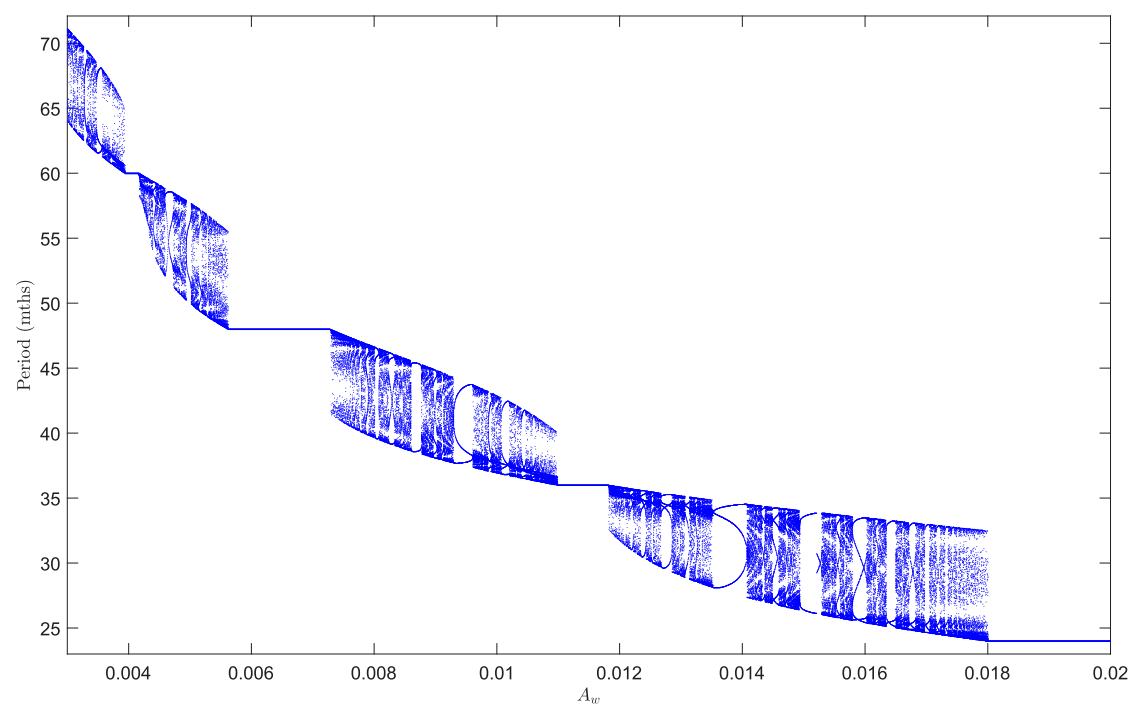

FIG. 5. Variation of QBO period with wave amplitude, for the Gompertz wave forcing profile. At each value of $A_{w}$, the set of measured QBO periods has been plotted.

top and bottom boundaries $z_{t}^{*}$ and $z_{b}^{*}$, respectively. For simplicity of presentation, we have assumed that the dissipation and diffusion parameters are constant in height, and we ignore density stratification; the derivation extends immediately to these cases as required.

We nondimensionalize (6) by introducing $H^{*}=$ $6.5 \mathrm{~km}, T^{*}=1$ day, and a horizontal velocity scale $U^{*}=c^{*}=30 \mathrm{~m} \mathrm{~s}^{-1}$. Thus, we can define nondimensional time, velocity, and height variables via

$$
t^{*}=T^{*} t, \quad \bar{u}^{*}=c^{*} \bar{u}, \quad \text { and } \quad z^{*}=17+H^{*} z .
$$

The model boundaries are nondimensionalized in a similar way:

$$
\left\{z_{b}^{*}, z_{t}^{*}\right\}=17+H^{*}\left\{z_{b}, z_{t}\right\}
$$

Substituting into (6) and rearranging gives the nondimensional HL equation

$$
\begin{aligned}
\frac{\partial \bar{u}}{\partial t}= & -w \frac{\partial \bar{u}}{\partial z}+\Lambda \frac{\partial^{2} \bar{u}}{\partial z^{2}} \\
& +\frac{F}{(\bar{u}-1)^{2}} \exp \left[-\int_{0}^{z} \frac{\gamma}{(\bar{u}-1)^{2}} d z\right] \\
& -\frac{F}{(\bar{u}+1)^{2}} \exp \left[-\int_{0}^{z} \frac{\gamma}{(\bar{u}+1)^{2}} d z\right]
\end{aligned}
$$

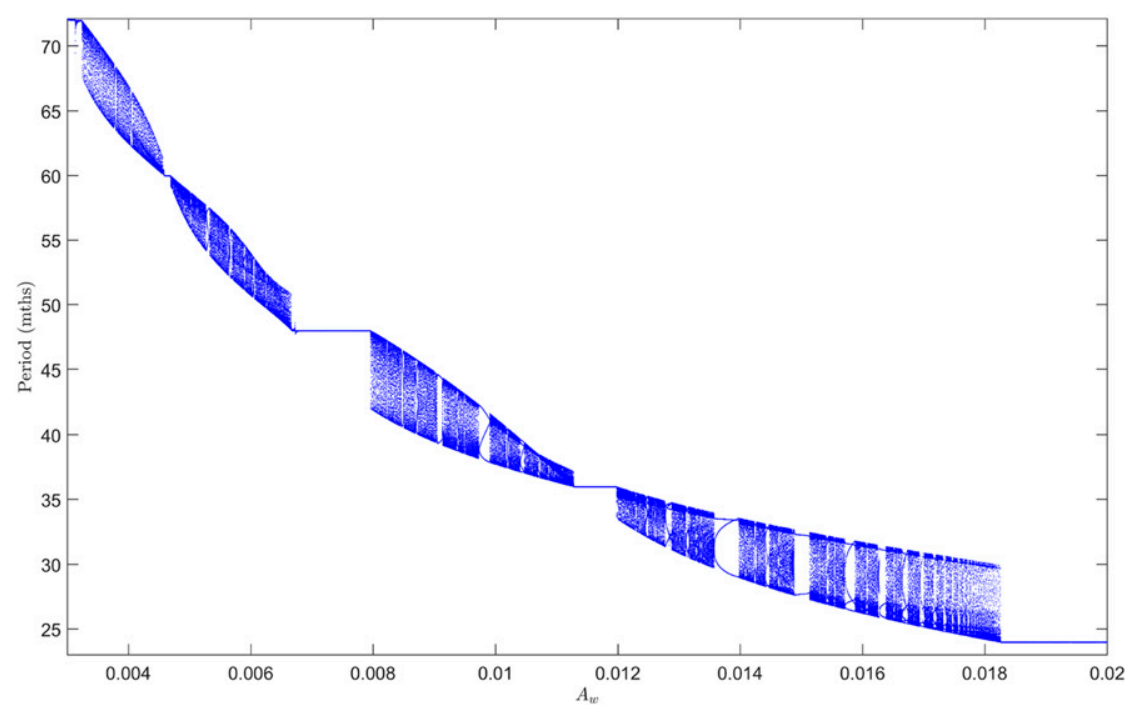

FIG. 6. As in Fig. 5, but for the sine wave amplitude profile. 
TABLE 1. Standard reference parameters used in the HL equation $[(6)]$.

\begin{tabular}{cll}
\hline \hline Parameter & \multicolumn{1}{c}{ Description } & \multicolumn{1}{c}{ Value } \\
\hline$z_{t}^{*}$ & Model upper boundary & $43 \mathrm{~km}$ \\
$z^{*}$ & Model lower boundary & $17 \mathrm{~km}$ \\
$N^{*}$ & Buoyancy frequency & $2.1 \times 10^{-2} \mathrm{~s}^{-1}$ \\
$\Lambda_{0}^{*}$ & Diffusion & $0.3 \mathrm{~m}^{2} \mathrm{~s}^{-1}$ \\
$k_{0}^{*}$ & Wave number & $2 \pi / 40000 \mathrm{~km}^{-1}$ \\
$c^{*}$ & Wave speed & $30 \mathrm{~m} \mathrm{~s}^{-1}$ \\
$\mu_{0}^{*}$ & Dissipation & $10^{-6} \mathrm{~s}^{-1}$ \\
\hline
\end{tabular}

where the nondimensional parameters $w, F, \gamma$, and $\Lambda$ are defined as

$$
\begin{gathered}
w=\frac{w * T *}{H *}, \quad F=\frac{F * N * \mu * T *}{k * c^{* 3}}, \\
\gamma=\frac{N * \mu * H *}{k^{*} c^{* 2}}, \quad \text { and } \quad \Lambda=\frac{\lambda_{0}^{*} T^{*}}{H^{* 2}} .
\end{gathered}
$$

We note that $\gamma \approx 1$ for the chosen parameters, and so for simplicity we will fix $\gamma=1$ in (9).

To convert the HL equation [(9)] into descent rate form we proceed as follows. Figure 7 shows a schematic diagram of the geometry of a surface of constant $\bar{u}$ across height $z$ and time $t$. We have marked the three points $A, B$, and $C$ on the diagram; these have coordinates $(z, t),(z, t+\Delta t)$, and $(z+\Delta z, t+\Delta t)$, respectively, where $\Delta z$ and $\Delta t$ are small. Points $A$ and $C$ sit on the constant $\bar{u}$ surface, and $B$ is a neighboring point. The wind field at point $C$ can be related to the wind field at point $A$ by going via point $B$ as follows:

$$
\begin{aligned}
\bar{u}(z+\Delta z, t+\Delta t)= & \bar{u}(z, t) \\
& +\bar{u}(z, t+\Delta t)-\bar{u}(z, t) \\
& +\bar{u}(z+\Delta z, t+\Delta t)-\bar{u}(z, t+\Delta t) .
\end{aligned}
$$

However, since both points $A$ and $C$ are on a surface of constant $\bar{u}$, we must have that $\bar{u}(z+\Delta z, t+\Delta t)=\bar{u}(z, t)$. Substituting this into (11) and rearranging gives

$$
\begin{aligned}
\frac{\bar{u}(z, t+\Delta t)-\bar{u}(z, t)}{\Delta t}= & -\frac{\bar{u}(z+\Delta z, t+\Delta t)-\bar{u}(z, t+\Delta t)}{\Delta z} \\
& \times \frac{\Delta z}{\Delta t} .
\end{aligned}
$$

Taking the limit as $\Delta t \rightarrow 0$ then yields

$$
\left.\frac{\partial \bar{u}}{\partial t}\right|_{z}=-\left.\left.\frac{\partial \bar{u}}{\partial z}\right|_{t} \frac{\partial z}{\partial t}\right|_{\bar{u}}
$$

The equation reveals that zonal wind acceleration and vertical descent can be related via vertical shear. By

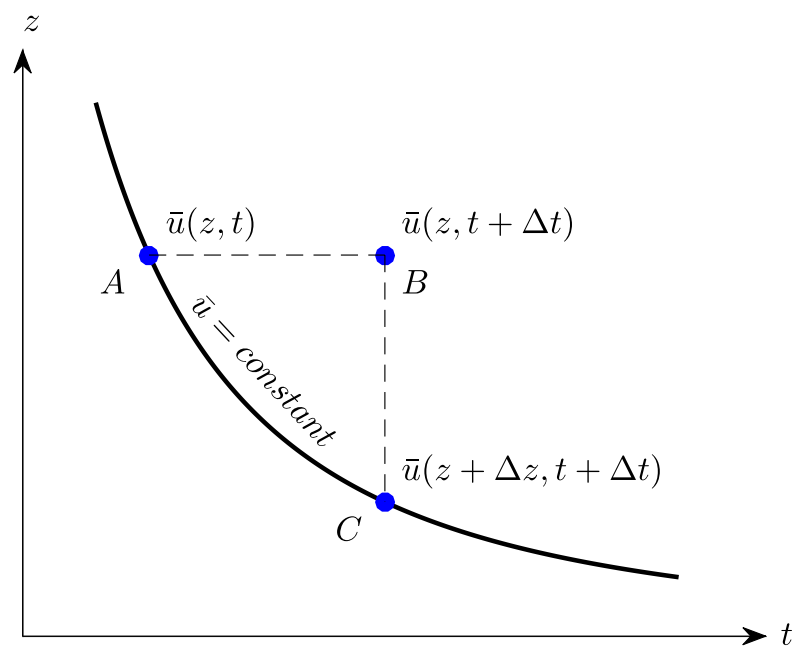

FIG. 7. Schematic for derivation of the relationship between horizontal acceleration and vertical descent. See text for details.

substituting (13) into the HL equation [(9)] and by setting the surface of constant $\bar{u}$ to be the zero wind surface $\bar{u}=0$, we can rearrange for $d z_{0} / d t=\partial z /\left.\partial t\right|_{\bar{u}=0}$. This gives the following descent rate model for the HL equation:

$$
\begin{aligned}
\frac{d z_{0}}{d t}= & w_{c}+w_{a} \cos \left(\frac{2 \pi t}{360}\right)-\left.\Lambda \frac{\partial^{2} \bar{u}}{\partial z^{2}}\right|_{z=z_{0}} /\left.\frac{\partial \bar{u}}{\partial z}\right|_{z=z_{0}} \\
& -F \exp \left[-\int_{0}^{z_{0}} \frac{1}{(\bar{u}-1)^{2}} d z\right] /\left.\frac{\partial \bar{u}}{\partial z}\right|_{z=z_{0}} \\
& +F \exp \left[-\int_{0}^{z_{0}} \frac{1}{(\bar{u}+1)^{2}} d z\right] /\left.\frac{\partial \bar{u}}{\partial z}\right|_{z=z_{0}}
\end{aligned}
$$

Here we have expressed upwelling $w$ as a sum of mean and annually varying parts $w_{c}$ and $w_{a}$, respectively. Note that the upwelling terms are of the exact same form as was posited in the heuristic model [(1)]; however, the forms of diffusion and wave forcing still depend on the velocity profile $\bar{u}$ and its derivatives at and below the height of the zero wind line.

To make further progress, we consider a specific form for $\bar{u}$. We assume that $\bar{u}$ is given by a quadratic function of height up to the zero wind line:

$$
\bar{u}(z)=\alpha_{2} z^{2}+\alpha_{1} z+\alpha_{0},
$$

for $0 \leq z \leq z_{0}$. The profile is subject to the following constraints:

$$
\bar{u}(0)=u_{0}, \quad \bar{u}\left(z_{0}\right)=0, \quad \text { and } \quad \frac{d \bar{u}}{d z}(0)=0 .
$$


The first two conditions simply state the values of the velocity profile at the heights of the lower boundary $(z=0)$ and the zero wind line $\left(z=z_{0}\right)$, respectively. The tropopause velocity $u_{0}$ was defined in Fig. $1 \mathrm{~b}$; its dynamics will be discussed in the next section. The third condition is a no-shear boundary condition that is applied at the lower boundary, following R16. We did not define a velocity profile for $z>z_{0}$, as wave dissipation above $z=z_{0}$ does not affect the dynamics at $z_{0}$ in the HL model (Plumb 1977).

The boundary conditions [(16)] are used to fix the values of the three parameters $\alpha_{1,2,3}$ in (15), giving the following form for the velocity profile for $0 \leq$ $z \leq z_{0}$ :

$$
\bar{u}(z)=u_{0}\left[1-\left(\frac{z}{z_{0}}\right)^{2}\right] .
$$

The zonal wind profile given above is in good qualitative agreement with the winds generated by the HL model below the zero wind line. The profile captures the progressive reduction in wind velocity as $z_{0}$ is approached from below while also allowing for the strength of the diffusion term at the zero wind line to increase as it descends. This latter feature is an important enabler of the collapse of shear zones at the tropopause in the HL model. A failure of this wind profile, however, is that it does not accurately reflect the strong wind shears present near the zero wind line. As a result, the diffusion term in the descent model is relatively weak as compared to the diffusion term in the full HL model.

Using (17), we now compute spatial derivatives of the zonal wind to yield

$$
\frac{\partial \bar{u}}{\partial z}=-\frac{2 u_{0}}{z_{0}^{2}} z, \quad \frac{\partial^{2} \bar{u}}{\partial z^{2}}=-\frac{2 u_{0}}{z_{0}^{2}}, \quad \text { and } \quad \frac{\partial^{2} \bar{u}}{\partial z^{2}} / \frac{\partial \bar{u}}{\partial z}=\frac{1}{z} .
$$

These expressions are then substituted into (14) and simplified. For the quadratic velocity profile [(17)] the integrals in (14) can also be evaluated analytically to give closed form expressions for the wave forcing; these are stated below.

The final equation for the descent rate is

$$
\frac{d z_{0}}{d t}=w_{c}+w_{a} \cos \left(\frac{2 \pi t}{360}\right)-\frac{\Lambda}{z_{0}}-F G\left(z_{0}, u_{0}\right),
$$

where the wave forcing $G\left(z_{0}, u_{0}\right)$ now depends on both $z_{0}$ and $u_{0}$, and is given by

$$
G\left(z_{0}, u_{0}\right)=\frac{z_{0}}{2 u_{0}}\left[e^{-N_{e}\left(z_{0}, u_{0}\right)}-e^{-N_{w}\left(z_{0}, u_{0}\right)}\right]
$$

with

$$
N_{w}\left(z_{0}, u_{0}\right)= \begin{cases}\frac{z_{0}}{2\left(1-u_{0}\right)}\left\{1-\frac{1}{2 \sqrt{u_{0}\left(u_{0}-1\right)}} \ln \left[\frac{\sqrt{u_{0}\left(u_{0}-1\right)}+u_{0}}{\sqrt{u_{0}\left(u_{0}-1\right)}-u_{0}}\right]\right\}, & u_{0}<0, \\ z_{0}, & u_{0}=0, \\ \frac{z_{0}}{2\left(1-u_{0}\right)}\left\{1-\frac{1}{\sqrt{-u_{0}\left(u_{0}-1\right)}} \arctan \left[\frac{-u_{0}}{\sqrt{-u_{0}\left(u_{0}-1\right)}}\right]\right\}, & u_{0}>0,\end{cases}
$$

and

$$
N_{e}\left(z_{0}, u_{0}\right)= \begin{cases}\frac{z_{0}}{2\left(u_{0}+1\right)}\left\{1+\frac{1}{\sqrt{-u_{0}\left(u_{0}+1\right)}} \arctan \left[\frac{-u_{0}}{\sqrt{-u_{0}\left(u_{0}+1\right)}}\right]\right\}, & u_{0}<0, \\ \frac{z_{0},}{2\left(u_{0}+1\right)}\left\{1+\frac{1}{2 \sqrt{u_{0}\left(u_{0}+1\right)}} \ln \left[\frac{\sqrt{u_{0}\left(u_{0}+1\right)}+u_{0}}{\sqrt{u_{0}\left(u_{0}+1\right)}-u_{0}}\right]\right\}, & u_{0}>0 .\end{cases}
$$


We note that $N_{w}\left(z_{0},-u_{0}\right)=N_{e}\left(z_{0}, u_{0}\right)$ and hence that $G\left(z_{0}, u_{0}\right)$ is even in $u_{0}$ : that is, $G\left(z_{0},-u_{0}\right)=G\left(z_{0}, u_{0}\right)$. This reflects the symmetry in the characteristics of the waves we have chosen.

\section{b. Dynamics of $u_{0}$}

To complete the description of the descent rate model, we need an equation for $u_{0}$. The evolution of $u_{0}$ can be deduced by discretizing the nondimensional HoltonLindzen equation [(9)] into $n$ uniformly spaced vertical levels $Z_{-1}, Z_{0}, Z_{1}, \ldots, Z_{n-2}$, with the separation between adjacent levels given by $h=\left(z_{t},-z_{b}\right) / n$. The corresponding zonal velocities at each height level are given by $u_{-1}, u_{0}, u_{1}$, $\ldots, u_{n-2}$. We then assume that the dynamics of the lower boundary are well captured by considering the evolution of the velocity field $u_{0}$ at the second grid point, $Z_{0}$.

If we approximate the spatial derivatives of (9) using their discrete counterparts, we can write the evolution equation of $u_{0}$ as follows:

$$
\begin{aligned}
\frac{d u_{0}}{d t}= & -w \frac{u_{1}-u_{0}}{h}+\Lambda \frac{u_{1}-2 u_{0}+u_{-1}}{h^{2}} \\
& +\frac{F}{\left(u_{0}-1\right)^{2}} \exp \left\{-\frac{1}{2}\left[\frac{h}{\left(u_{0}-1\right)^{2}}+\frac{h}{\left(u_{-1}-1\right)^{2}}\right]\right\} \\
& -\frac{F}{\left(u_{0}+1\right)^{2}} \exp \left\{-\frac{1}{2}\left[\frac{h}{\left(u_{0}+1\right)^{2}}+\frac{h}{\left(u_{-1}+1\right)^{2}}\right]\right\} .
\end{aligned}
$$

Here we have approximated the integral using the trapezium rule.

We note that the no-shear boundary condition implies that $u_{-1}=u_{0}$. Further, we identify $u_{1}$ with $\bar{u}(h)=u_{0}\left(1-h^{2} / z_{0}^{2}\right)$ where $\bar{u}$ refers to the quadratic approximation of the zonal wind [(17)] and recall that $z_{0}$ is the height of the zero wind line. Substituting these values into (23) and simplifying yields the following evolution equation for the dynamics of $u_{0}$ :

$$
\begin{aligned}
\frac{d u_{0}}{d t}= & -\frac{\Lambda^{\prime}}{z_{0}^{2}} u_{0}+\frac{F}{\left(u_{0}-1\right)^{2}} \exp \left\{-\frac{h}{\left(u_{0}-1\right)^{2}}\right\} \\
& -\frac{F}{\left(u_{0}+1\right)^{2}} \exp \left\{-\frac{h}{\left(u_{0}+1\right)^{2}}\right\},
\end{aligned}
$$

where

$$
\Lambda^{\prime}=\Lambda-w h
$$

represents the combined effects of diffusion and upwelling.
The essential dynamics of (24) are summarized in Figs. $8 \mathrm{a}-\mathrm{c}$ for $h=0.02$ (corresponding to a separation distance of $150 \mathrm{~m}$ between height levels) and $F=h e$. This value of $F$ was chosen in order to normalize the wave forcing profiles in Fig. 8. Each plot displays graphs of the total wave forcing (solid line) and the negative of the total resistance $-\Lambda^{\prime} u_{0} / z_{0}^{2}$ (dashed line), plotted against the possible values of $u_{0}$. The points where the lines intersect give the equilibrium values of $u_{0}$; stable and unstable equilibria are denoted by filled and open circles, respectively.

The three plots represent the changing force balance between the waves and diffusion/upwelling over the course of descent of a zero wind line. While the value of the wave forcing at the tropopause is independent of the position of $z_{0}$, the total resistance is not and in fact increases as the zero wind line descends. Figure 8a depicts the situation when a new shear layer forms in the upper levels of the model. The large value of $z_{0}$ is associated with a correspondingly weak resistance term at the lower boundary, as reflected in the shallow gradient of the dashed black line. There are three equilibria: an unstable origin and two stable points close to the extremal values of $u_{0}= \pm 1$. The value of $u_{0}$ is quickly attracted to one of the extremal equilibria, as determined by the initial sign of $u_{0}$.

As the shear zone descends, the resistance term $-\Lambda^{\prime} u_{0} / z_{0}^{2}$ increases in strength, and the gradient of the dashed line steepens. Two new unstable equilibria are created in a subcritical pitchfork bifurcation, which also stabilizes the origin (Fig. 8b). Since the value of $u_{0}$ would have been closer to the extremal stable equilibria at the onset of the bifurcation, however, $u_{0}$ will continue to track the location of one of the extremal stable equilibria.

Finally, when the zero wind line is close to the bottom boundary (Fig. 8c), the resistance increases to the point where the stable extremal equilibria collide with the previous unstable equilibria and are annihilated in a saddle-node bifurcation. This is the point in the QBO evolution when the zonal winds at the lower boundary are attracted toward the origin and so begin to decelerate and eventually switch direction. Our quadratic approximation to the zonal wind field no longer holds through the transition.

To step the model forward at this point, we make the assumption that a new shear zone forms aloft as soon as $z_{0}$ descends to the height where the saddle-node bifurcation occurs. Thus, the bifurcation point becomes a trigger at which we impose $z_{0}=4$ (the upper boundary of the model). This change in height of $z_{0}$ immediately reduces the strength of the resistance force at the lower boundary, and the system returns to the situation described in Fig. 8a but now with $u_{0}$ attracted to the opposite stable equilibrium than it was before. 
(a)

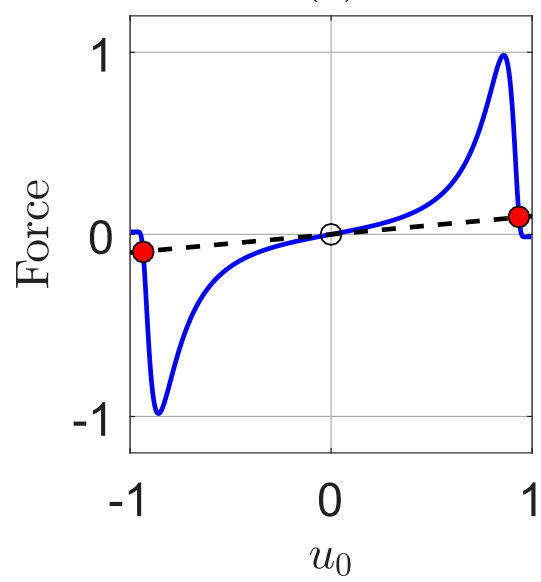

(b)

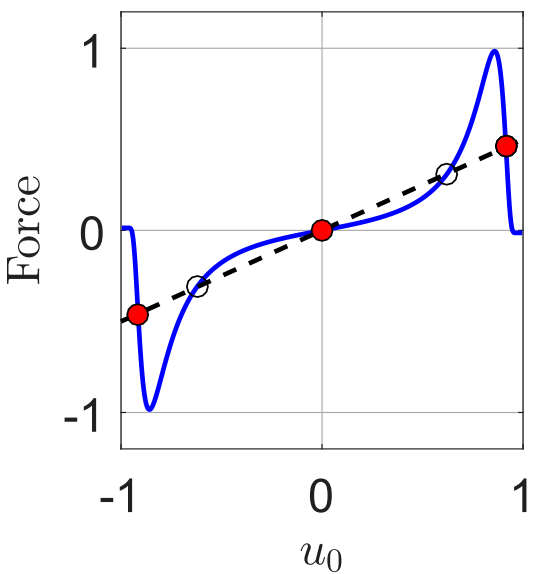

(c)

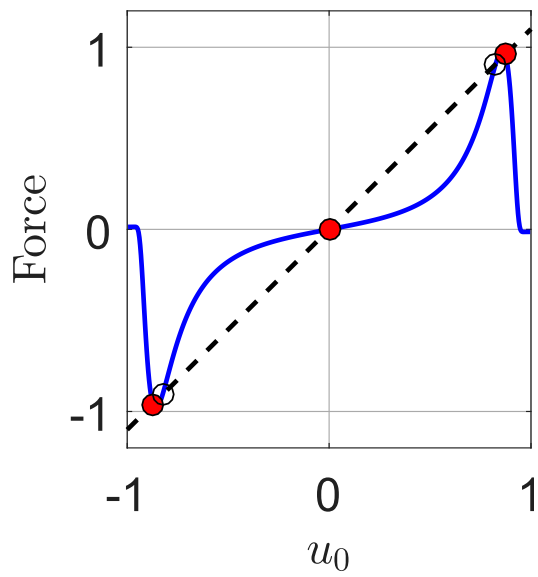

FIG. 8. Changes in the stable and unstable equilibria at the lower boundary over the course of descent of a shear zone. The net wave forcing at the lower boundary (solid line) and the negative of the sum of diffusive and upwelling forces at the lower boundary (dashed line) are shown; see the first term on the right-hand side of (24). Stable (filled circle) and unstable (open circle) equilibria are indicated.

We can derive an expression for the bifurcation height—call it $z_{c}$-by approximating the wave forcing terms by simple linear functions for values of $u_{0}$ near to \pm 1 ; see Fig. 9. Each peak in the wave forcing profile corresponds to the point of maximum forcing driven by either the westerly (positive peak) or easterly (negative peak) wave-the individual wave contributions are depicted by the dashed blue lines in Fig. 9. For small values of $h$, the contribution to the peak forcing is given almost entirely by a single wave, and so we can calculate the location of each peak $u_{0}^{ \pm}$by setting

$$
\begin{aligned}
& \frac{d}{d z}\left\{\frac{F}{\left(u_{0}^{-}-1\right)^{2}} \exp \left[-\frac{h}{\left(u_{0}^{-}-1\right)^{2}}\right]\right\}=0, \\
& \frac{d}{d z}\left\{\frac{F}{\left(u_{0}^{+}+1\right)^{2}} \exp \left[-\frac{h}{\left(u_{0}^{+}+1\right)^{2}}\right]\right\}=0,
\end{aligned}
$$

where $u_{0}^{ \pm}$are the values of $u_{0}$ for which the corresponding wave forcings are largest. Solving (26) yields $u_{0}^{-}=-1+\sqrt{h}$ and $u_{0}^{+}=1-\sqrt{h}$, with corresponding peak wave forcings of $\pm F / h e$ respectively.

We can fit straight lines through the peak wave forcing values that also pass through $u_{0} \pm 1$ as appropriate (these are the dash-dotted black lines in Fig. 9). In this approximation we get the following expressions for $u_{0}$ dynamics near the turning points:

$$
\begin{aligned}
& \frac{d u_{0}}{d t}=-\frac{F}{h^{3 / 2} e}\left(1+u_{0}\right)-\frac{\Lambda^{\prime}}{z_{0}} u_{0}, \quad u_{0}<0, \\
& \frac{d u_{0}}{d t}=\frac{F}{h^{3 / 2} e}\left(1-u_{0}\right)-\frac{\Lambda^{\prime}}{z_{0}} u_{0}, \quad u_{0}>0 .
\end{aligned}
$$

At the equilibrium points we can set $d u_{0} / d t=0$, which allows us to rearrange (27) and (28) to yield expressions for the equilibrium values of $u_{0}$ :

$$
u_{0}= \pm \frac{z_{0}^{2}}{z_{0}^{2}+\chi^{2}}= \pm \frac{1}{1+\frac{\chi^{2}}{z_{0}^{2}}}
$$

where

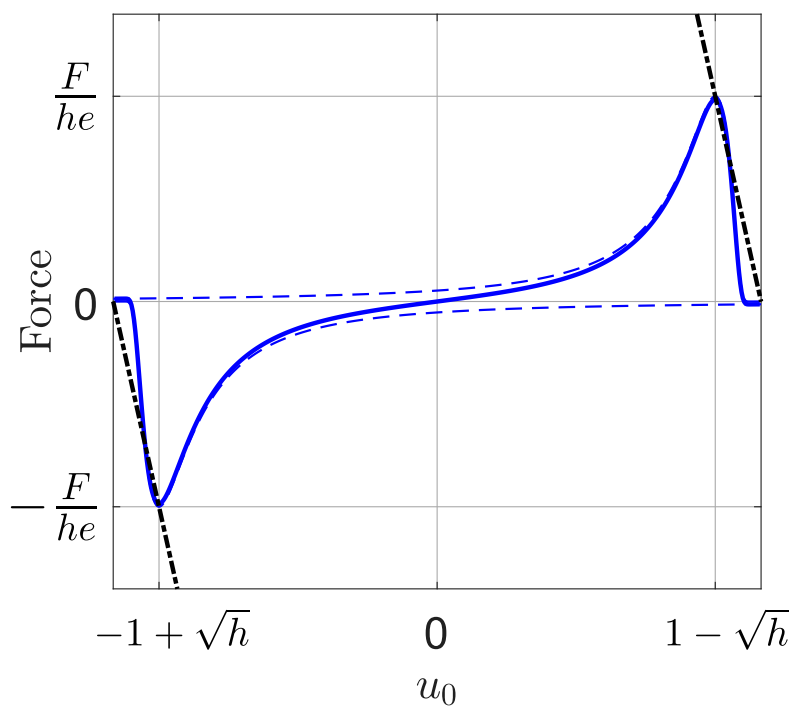

FIG. 9. Linear approximations to the wave forcing near the peak values of wave forcing. The wave forcing contributed by each wave for different values of $u_{0}$ (two dashed blue lines); the sum of these two wave forcings (solid blue line). Linear approximations to the wave forcing near the peak forcing values (dash-dotted black lines). 
(a)

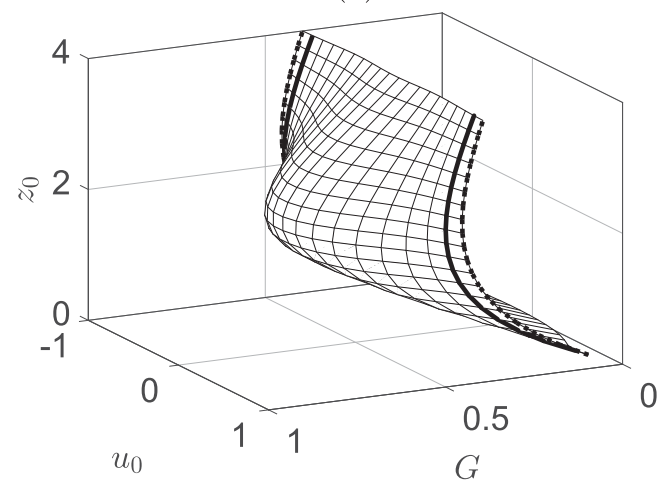

(b)

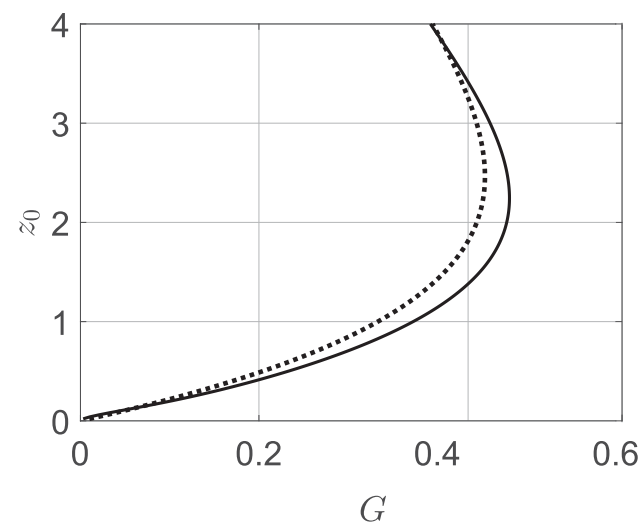

FIG. 10. (a) Profile of the wave forcing $G_{0}\left(z_{0}, u_{0}\right)$ as defined in (20)-(22) for the full HL descent rate model [(19)]. The lines give the values of $G$ within the bounds of the realizable values of $u_{0}$ in the model. (b) These lines are replotted in the vertical for positive values of $u_{0}$.

$$
\chi^{2}=\frac{\Lambda^{\prime} h^{3 / 2} e}{F} .
$$

As described earlier, for large values of $z_{0}$, the equilibrium value of $u_{0}$ is very close to either +1 or -1 , depending on the initial condition. As the shear layer descends, $z_{0}$ decreases and so the magnitude of $u_{0}$ also decreases.

At the bifurcation height $z_{c}$, we have that $u_{0}=-1+\sqrt{h}$ or $u_{0}=-1-\sqrt{h}$. Substituting these values into (27) and (28) and rearranging then yields a single expression for the bifurcation height:

$$
z_{c}=\sqrt{\frac{(\Lambda-w h)(1-\sqrt{h}) h e}{F}} .
$$

\section{c. Results}

Figures $10 \mathrm{a}$ and $10 \mathrm{~b}$ show the value of the wave forcing profile $G\left(z_{0}, u_{0}\right)$ as defined in (20), for different values of $z_{0}$ and $u_{0}$. The wave forcing has the shape of a paraboloid (Fig. 10a). Recalling our discussion in the previous section, however, we note that $u_{0}$ values are largely confined to stay within two quite narrow ranges of values of width $\sqrt{h}$ on the extreme ends of the $u_{0}$ range. The corresponding range of $G$ values within these bounds have been highlighted in Fig. 10a as the narrow strips bounded by the bold solid and dotted lines.

Figure $10 \mathrm{~b}$ plots the vertical slices through the solid and dotted lines corresponding to $u_{0}=-1-\sqrt{h}$ and $u_{0}=1$, respectively, in Fig. 10a. The two curves give the range of variation of the wave profile $G$ in the HL descent equation as the shear zone descends and $u_{0}$ varies. The profiles can be compared to the heuristic Gompertz and sine wave profiles described in (4) and
(5), respectively (see also Fig. 3). The wave profile in the full model is more similar to that of the sine profile than to the Gompertz profile. The main difference is the asymmetry of the HL wave profile; rather than weakening symmetrically from $z_{0}=2$ as in the sine profile, the wave forcing remains fairly significant up to the top of the model.

We solve the full descent model of the HL (19) numerically, using values for $u_{0}$ given by (29). The nondimensional parameters $w_{c}, w_{c}$, and $\Lambda$ are fixed by setting the corresponding dimensional quantities $w_{c}^{*}=0.3 \mathrm{~mm} \mathrm{~s}^{-1}$, $w_{a}^{*}=0.2 \mathrm{~mm} \mathrm{~s}^{-1}$, and $\Lambda_{0}^{*}=0.3 \mathrm{~m}^{2} \mathrm{~s}^{-1}$ respectively. The initial condition is taken to be the descent of a westerly shear zone, with the initial zero wind height given by $z_{0}(0)=3.5$. When the value of $z_{0}$ reaches the critical bifurcation height given by (31), the value of $z_{0}$ is immediately reset to $z_{0}=4$, simulating the destruction of the shear zone at the lower boundary and the creation of a new shear zone at the upper boundary.

Figure 11 displays results for a sample simulation with forcing wave amplitude set at $F^{*}=8.3 \times 10^{-2} \mathrm{~m}^{2} \mathrm{~s}^{-2}$, which corresponds to a nondimensional value of $F=$ 0.0036 . The zero wind line descends as expected and is a good approximation to the dynamics of the zero wind line of the QBO. The average period of the QBO was found to be 32.3 months. We note that the wave amplitude $F$ required to produce this period is very large, around 4 times the maximum value of $F$ utilized in R16.

Finally, we vary $F^{*}$ systematically across 5000 values between $F^{*}=5.0 \times 10^{-2}$ and $10.0 \times 10^{-2} \mathrm{~m}^{2} \mathrm{~s}^{-2}$ and measure the set of QBO periods that result for each value of $F^{*}$, as described previously at the end of section 2. Results are plotted in Fig. 12. The resulting variations in the QBO period are very similar to the heuristic model results especially that of the sine wave profile case (Fig. 6). Once 
(a)

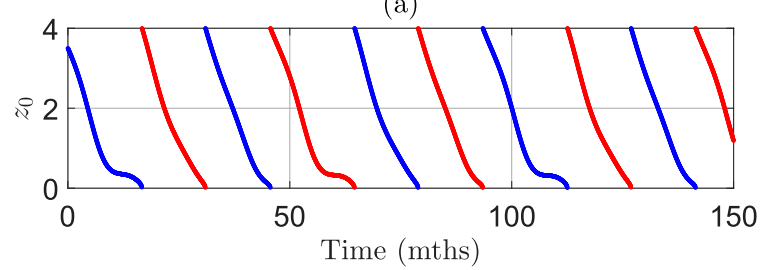

(b)

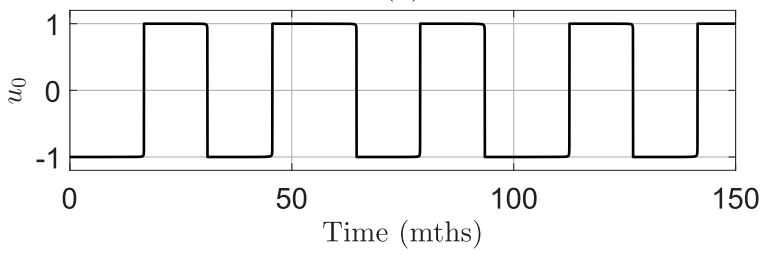

FIG. 11. (a) Sample solution of the HL descent rate model. Descending westerly (blue line) and easterly (red line) shear zones are indicated. (b) Corresponding evolution of $u_{0}$.

again we see all the hallmarks of synchronization, including locking regions, multiperiod cycles, and quasi periodicity.

\section{d. Connections to the circle map}

The set of behaviors described by the descent rate models are reminiscent of a class of mathematical models known as circle maps, which are well known in the literature of dynamical systems theory (Ott 2002; Jensen et al. 1984; Katok and Hasselblatt 1997). The classical "sine" circle map was defined by Arnold (1965) as a map on the circle:

$$
\theta_{n+1}=f\left(\theta_{n} ; \Omega, K\right)=\theta_{n}+\Omega-\frac{K}{2 \pi} \sin \left(2 \pi \theta_{n}\right) .
$$

In general, $f$ could be any periodic function in $\theta$, and we take $\Omega>0$ and $K \geq 0$. Here $f(\theta+1)=f(\theta)$, and $\theta \in[0,1]$. Geometrically, we can imagine "wrapping" the $\theta$ values onto a circle with a circumference of 1 . In the absence of nonlinearity $(K=0)$, the map represents uniform translation along the circle in increments of $\Omega$. For irrational $\Omega$, the orbit of the circle map is quasi periodic and will densely fill out the circle. However, if $\Omega$ is rational $-\Omega=p / q$, say, where $p$ and $q$ are integers sharing no common factorsthen the orbit will be periodic with period $q$.

Nonzero values of $K$ introduce nonlinearity into the system, resulting in a myriad of interesting new features. The dynamics can be captured by considering the winding number $W$, defined as

$$
W=\lim _{n \rightarrow \infty} \frac{f^{n}\left(\theta_{0}\right)-\theta_{0}}{n} .
$$

Here $f^{n}$ denotes the application of the map [(32)] $n$ times, and $\theta_{0}$ is the initial value. The winding number is independent of the choice of $\theta_{0}$, but it varies depending on the values of $\Omega$ and $K$.

For each value of $\Omega$ and $K$, the corresponding value of $W$ is either rational or irrational, reflecting either periodic or quasiperiodic motion. Arnold (1965) showed that for small values of $K$, the Lebesgue measure of the set of $\Omega$ values yielding irrational $W$ values is close to 1 ; that is, the orbit of the map is quasi periodic for almost all values of $\Omega$. He also demonstrated, however, that the distribution of these values is nontrivial in the sense that arbitrarily close to every value of $\Omega$ yielding quasi

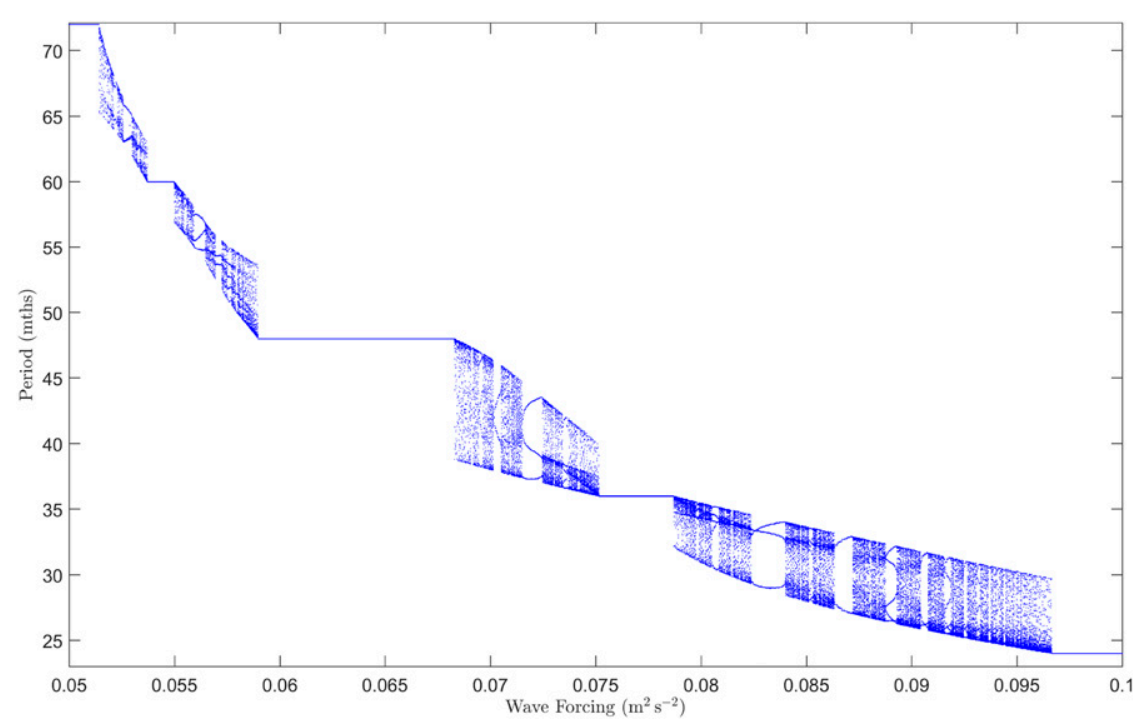

FIG. 12. Variation of QBO period with $F^{*}$, for the full HL descent rate model. At each value of $F^{*}$, the set of measured QBO periods has been plotted. 
(a)

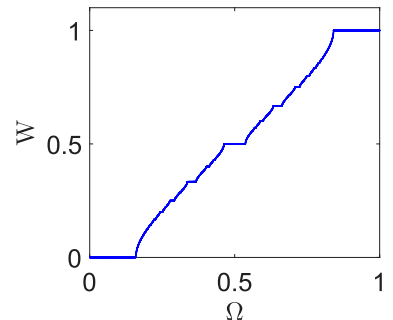

(b)

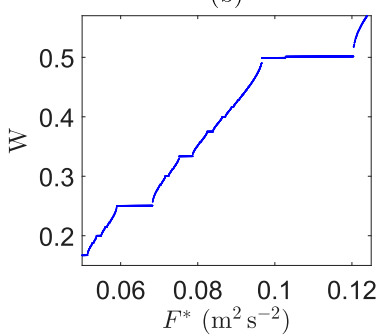

FIG. 13. Winding number variations in the case of (a) the circle map, and (b) the QBO generated by the HL descent rate model [(19)].

periodicity there is an interval of $\Omega$ values that yield rational winding numbers (periodic motion).

As the strength of $K$ increases, the lengths of the rational intervals increase and start to crowd out the quasiperiodic orbits. Eventually, when $K=1$, the set of $\Omega$ values yielding quasi periodicity is squeezed into a set of Lebesgue measuring zero, meaning that the map generates periodic motion for almost all values of $\Omega$. A plot of $W$ against $\Omega$ for the sine circle map is depicted in Fig. 13a, in the case when $K=1$. The resulting curve is a complex geometrical object known as a devil's staircase. The curve is a fractal and contains structure at all scales.

We can conceptually link our descent rate model of the HL equation to the circle map as follows. First, we define a transformation to map the different phases of the QBO onto an angle variable $\phi \in[0,1]$ :

$$
\phi\left(z_{0}, u_{0}\right)= \begin{cases}1-\frac{1}{2} \frac{z_{0}-z_{b}}{z_{t}-z_{b}}, & u_{0}<0, \\ \frac{1}{2}-\frac{1}{2} \frac{z_{0}-z_{b}}{z_{t}-z_{b}}, & u_{0}>0 .\end{cases}
$$

Hence, $\phi \in[0,1 / 2]$ during the descent of an easterly shear zone (i.e., when $u_{0}>0$ ), with $\phi=0$ when $z_{0}=z_{t}$ and $\phi=1 / 2$ when $z_{0}=z_{b}$. Similarly, $\phi \in[1 / 2,1]$ during the descent of a westerly shear zone. Next, we define time points $t_{n}=360(n-1)$ for $n \geq 1$, with the 360-day increment between time points being the period of tropical upwelling variation. Finally, by sequentially integrating (19) from time $t_{n}$ to time $t_{n+1}$ and transforming the resulting values of $z_{0}$ and $u_{0}$ into $\phi$ values via (34), we obtain a one-dimensional iterative map,

$$
\phi_{n+1}=\mathscr{F}\left(\phi_{n} ; \Lambda_{0}^{*}, w_{c}^{*}, w_{a}^{*}, F^{*}\right) .
$$

Here we have expressed the parameters in dimensional form. If we fix $w_{c}^{*}=0.3 \mathrm{~mm} \mathrm{~s}^{-1}, w_{a}^{*}=0.2 \mathrm{~mm} \mathrm{~s}^{-1}$, and $\Lambda_{0}^{*}=0.3 \mathrm{~m}^{2} \mathrm{~s}^{-1}$ as before, we can vary $F^{*}$ and calculate the corresponding variation in the winding number for the map [(35)]; this is depicted in Fig. 13b. The
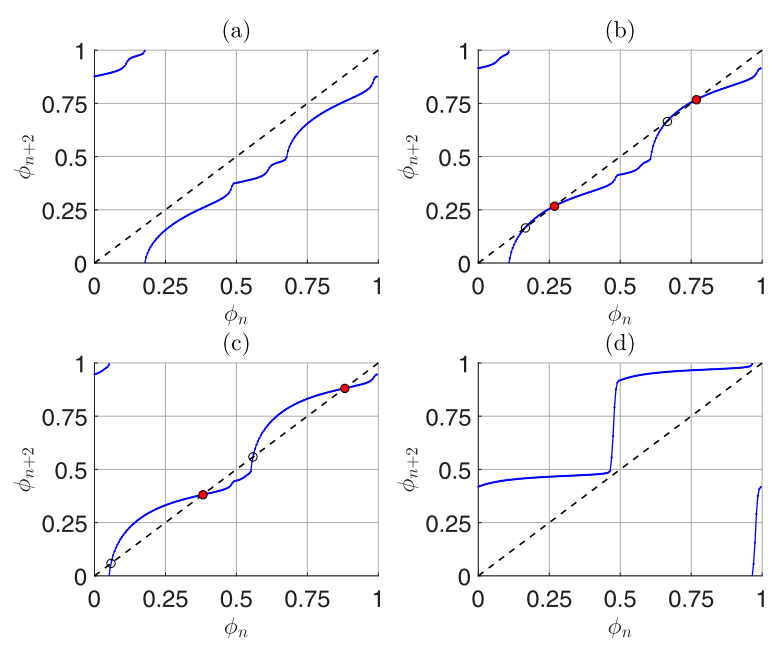

FIG. 14. Plots of the map $\phi_{n+2}=\mathscr{F}^{2}\left(\phi_{n}\right)$ for $F^{*}=$ (a) 0.091 , (b) 0.097 , (c) 0.103 , and (d) $0.127 \mathrm{~m}^{2} \mathrm{~s}^{-2}$. In each plot, the identity line $\phi_{n+2}=\phi_{n}$ has been plotted (black dashed line). Stable (red filled circle) and unstable (black open circle) fixed points are marked.

similarities between the results of iterating the descent model map and the circle map are striking; it is clear that the basic fractal structure of the staircase is present in the QBO model as well. Qualitatively similar plots were produced using both the Gompertz profile and the full descent model (not shown). Thus, it would seem reasonable to claim that that the mathematical structure of QBO descent in the presence of external periodic influences is essentially described by a circle map.

Analysis of the map [(35)] can help us to understand how locking regions are created. As an example, we consider the dynamics of the map as the system passes through the $W=0.5$ locking region. A value of $W=0.5$ indicates that the average progression of $\phi$ over the course of one map iteration is 0.5 , which implies the QBO completes a full cycle in two iterations of the map. We therefore consider the graph of $\phi_{n+2}=\mathscr{F}^{2}\left(\phi_{n}\right)$, which is presented in Figs. 14a-d for four different values of $F^{*}$ between 0.091 and $0.127 \mathrm{~m}^{2} \mathrm{~s}^{-2}$ (these values correspond to points in the $W=0.5$ locking region; refer to Fig. 13b). Fixed points of the map are located at the intersection of the map with the identity line $\phi_{n+2}=\phi_{n}$, which has been denoted by a dashed line in each figure.

When $F^{*}=0.091 \mathrm{~m}^{2} \mathrm{~s}^{-2}$ (Fig. 14a)—just before entering the locking region-the map $\mathscr{F}^{2}$ does not intersect the identity line and thus has no fixed points. As a result there is no 24-month frequency locking for this value of $F^{*}$. As $F^{*}$ increases, however, the graph of $\mathscr{F}^{2}$ shifts upward and eventually crosses the identity line at around $F^{*}=0.097 \mathrm{~m}^{2} \mathrm{~s}^{-2}$, creating stable and unstable fixed points in a tangent bifurcation (Fig. 14b). The two 
(a)

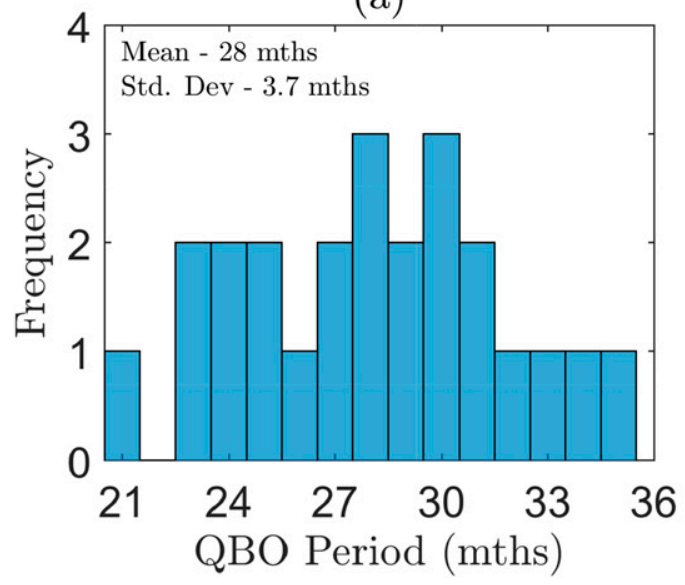

(b)

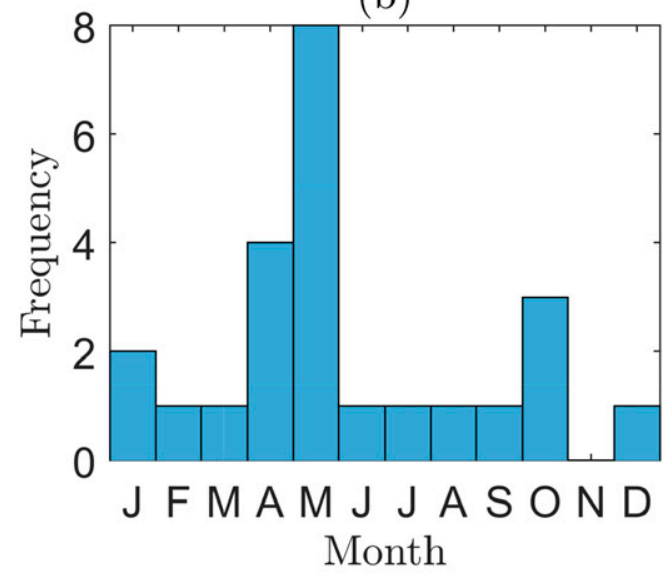

FIG. 15. (a) Histogram of QBO periods measured in the ERA data at $40 \mathrm{hPa}$. (b) Histogram of the westerly QBO onset month at $40 \mathrm{hPa}$.

stable fixed points are separated by exactly 0.5 , reflecting the symmetry of the assumed wave forcings in the model. The existence of the attracting fixed points means that as the map $\mathscr{F}^{2}$ is iterated forward, all points in the domain (excepting the two unstable fixed points) are attracted to one of the two stable fixed points. Thus, with repeated iterations of the map, the QBO will eventually enter a state where it completes one full cycle every 2 years, regardless of its starting phase.

As $F^{*}$ increases further, the QBO will remain frequency locked to the 24-month cycle so long as the fixed points of $\mathscr{F}^{2}$ persist (Fig. 14c). Eventually, however, as the graph of $\mathscr{F}^{2}$ continues to rise and deform with increasing $F^{*}$, the fixed points are destroyed in an inverse tangent bifurcation (Fig. 14d). The curve then no longer intersects the identity line, and so the $W=0.5$ frequency locking region ends.

\section{Observational evidence for multicycle QBO structure}

We now investigate whether the clustering of QBO periods under synchronization-so clearly observed in both the HL model and the descent rate modelsis in fact observed in the real atmosphere. To this end we analyzed monthly mean zonal-mean zonal wind data from atmospheric reanalysis datasets from the European Centre for Medium-Range Weather Forecasts (ECMWF), consisting of the ERA-40 data (Uppala et al. 2005) covering the period 1957-78 and the ERA-Interim data (Dee et al. 2011) for the period 19792015. We considered data only up to August 2015, to avoid complications arising from the disruption of the QBO in late 2015 and 2016 (Newman et al. 2016; Osprey et al. 2016).
Monthly zonal-mean zonal wind data were extracted and averaged over the latitudes of $5^{\circ} \mathrm{S}-5^{\circ} \mathrm{N}$. We converted the model pressure levels $p$ to log-pressure $z$ using

$$
z(p)=H \ln \left(\frac{1000 \mathrm{hPa}}{p}\right)
$$

where an atmospheric scale height of $H=7.64 \mathrm{~km}$ was assumed. The location of the westerly onset zero wind line over time was then estimated by a linear interpolation of the velocity field between the heights across which the velocity changed sign from easterly to westerly. Finally, the periods of the QBO cycles were estimated by measuring the times between westerly zero wind line crossings at $40 \mathrm{hPa}(z=24.6 \mathrm{~km})$. This choice of height level is somewhat arbitrary; we found consistent results for all height levels between 24 and $29 \mathrm{~km}$ (not shown).

We identified 24 complete QBO cycles over the selected period. The mean period of these cycles was 28 months, with a standard deviation of 3.7 months. A histogram of QBO periods is displayed in Fig. 15a. The distribution is quite flat and has a fairly large spread, with cycle lengths as low as 21 months and as high as 35 months. The seasonal tendency of the QBO is highlighted in Fig. 15b, which shows a histogram of the onset months of the westerly QBO at $40 \mathrm{hPa}$. There is a clear preference for westerly QBO onsets to occur in April and May.

We wished to interpret the abovementioned seasonal tendency of QBO onsets in terms of synchronization by the annual cycle. To this end, we separated the time series of observed QBO periods into 10 distinct periods, 
TABLE 2. Separation of the observed QBO periods in ERA data into distinct time intervals (first column), with each time interval consisting of an exact number of complete QBO cycles. The lengths of the constituent periods (months) are listed in the second column and their sum is listed in the third column.

\begin{tabular}{cccccc}
\hline \hline Period & \multicolumn{4}{c}{ Individual QBO periods } & Row sum \\
\hline Aug 1959-May 1961 & 21 & & & 21 \\
May 1961-May 1969 & 29 & 33 & 34 & 96 \\
May 1969-May 1971 & 24 & & & & 24 \\
May 1971-May 1980 & 23 & 27 & 30 & 28 & 108 \\
May 1980-May 1990 & 29 & 30 & 30 & 31 & 120 \\
May 1990-Apr 1997 & 31 & 25 & 28 & & 83 \\
Apr 1997-Apr 2004 & 23 & 35 & 26 & 84 \\
Apr 2004-May 2008 & 25 & 24 & & 49 \\
May 2008-May 2013 & 28 & 32 & & & 60 \\
May 2013-Aug 2015 & 27 & & & & 27 \\
\hline
\end{tabular}

each consisting of an exact number of QBO cycles as measured at $40 \mathrm{hPa}$. The makeup of each interval is shown in Table 2, with the columns of the table denoting the time interval, the length of individual QBO cycles within that time interval, and the sum of the QBO periods within the time interval, respectively. It is immediately clear from the table that the QBO periods within each period sum to a multiple of 12 months (with an error margin of \pm 1 month). The only exceptions are the first and final periods, which are possibly incomplete. These observations are clearly consistent with our earlier findings, as well as the findings of R16, that the QBO tends to cluster into sets that add to a multiple of the forcing period as a result of its interaction with the annual cycle in tropical upwelling.

To test whether the abovementioned clustering of QBO periods is statistically significant, we computed the vector of cumulative sums $\mathbf{C}$ of the $\mathrm{QBO}$ periods, for which the $n$th entry is defined as $C_{n}=\sum_{i=1}^{n} p_{i}$. Here $p_{j}$ is the period of the $j$ th $\mathrm{QBO}$ cycle and $n$ ranges from 1 to the total number of QBO periods in the dataset. We then calculate the vector of remainders $R_{n} \equiv C_{n}$ $(\bmod 12)$, which is the set of remainders when each cumulative sum $C_{n}$ is divided by 12 .

If successive sets of $\mathrm{QBO}$ periods tend to add to multiples of 12 , then we would expect a peak to form in the histogram of $R_{n}$ as calculated over the whole dataset. For example, consider a set of periods $p_{j}=\{17,22,26,29,32,23\}$, which is made up a single period of length 17 , followed by a twocycle period of length 48 and a three-cycle period of length 84 . The corresponding set of remainders is $R_{n}\{5,3,5,10$, $6,5\}$; the histogram of $R_{n}$ has a peak at $R_{n}=5$ because of the two-cycle and three-cycle periods, thus reflecting the influence of the annual cycle on the QBO periods.

Figure 16a shows the histogram of $R_{n}$ for the QBO periods measured in the ERA datasets. There are strong peaks and troughs in the bin heights, consistent with the clustering of QBO periods as a result of synchronization to the 12-month annual cycle. We note that the histogram of $R_{n}$ is closely related to the QBO onset month histogram depicted in Fig. 15b; there is a close correspondence if we associate bin 0 with the onset month of the first QBO cycle and translate the other months accordingly (the lack of an exact correspondence is due to the rounding errors introduced when assigning onset months). This provides a conceptual link between these two seemingly separate observations; a large peak in the QBO onset month histogram represents the same phenomenon as the strong clustering tendency of the QBO periods, and both of these are indicators of synchronization to the annual cycle.

We measured the spread of the bin heights in Fig. 16a by calculating their standard deviation: $\sigma=1.95$. The statistical significance of this spread in bin heights was tested by generating 10000 sets of simulated QBO periods. Each set was generated by repeatedly drawing from a normal distribution with the mean and standard deviation given by the mean and standard deviation, respectively, of the observed QBO periods in the ERA data. For consistency, the number of periods drawn for each simulated set was the same as the number of full QBO cycles observed in the data.

For each of the 10000 sets, a corresponding histogram of the remainders $R_{n}$ was produced and the standard deviation of the bin heights measured. This yielded a set of 10000 standard deviation values. Figure $16 \mathrm{~b}$ shows the probability-normalized histogram of these bin height standard deviations. This distribution was used to calculate the probability of occurrence of any given spread of bin heights as predicted by the null hypothesis; in particular, we measured the probability of observing a bin height standard deviation that was at least as extreme as that found in the ERA dataset. As noted in Fig. 16b, the probability of observing a bin height standard deviation equal to or greater than observed is $4 \%$. This provides sufficient evidence (at the 5\% significance level) to reject the hypothesis that the observed clustering of the QBO periods is simply caused by random fluctuations.

\section{Discussion}

The descent rate models introduced in this paper provide a powerful framework within which to interpret synchronization of the QBO. The essential dynamics are captured in a single ordinary differential equation; this represents a huge reduction in complexity from the Holton-Lindzen equation, which is a nonlinear secondorder partial differential equation. We have also demonstrated that descent models are closely related to the circle map, a mathematical model of frequency locking 
(a)

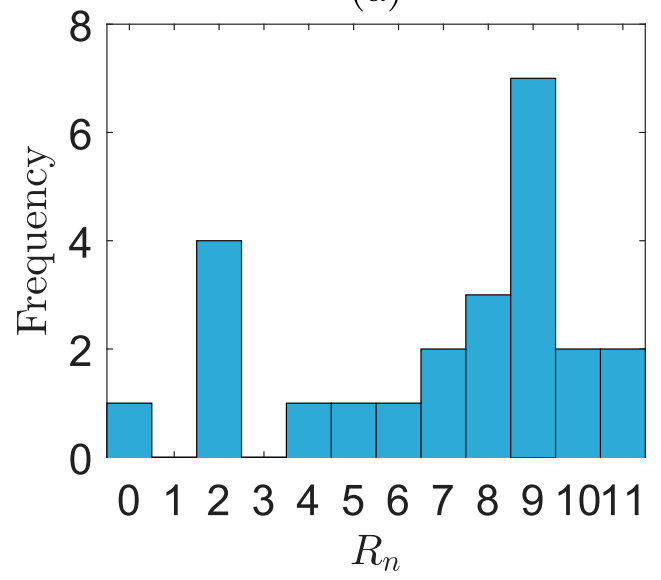

(b)

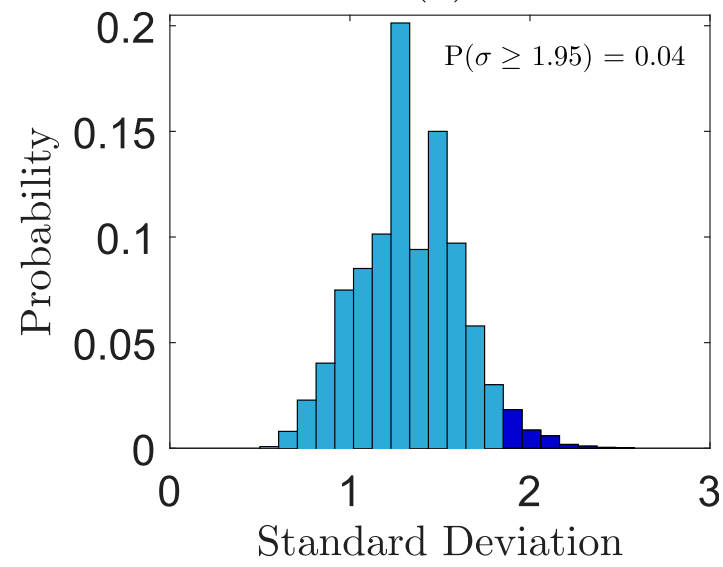

FIG. 16. (a) Histogram of $R_{n} \equiv C_{n}(\bmod 12)$, where $C_{n}=\sum_{i=1}^{n} p_{i}$ is the cumulative sum of the first $n$ QBO periods. (b) Corresponding derived probability distribution based on a null hypothesis that the QBO period is normally distributed. The darkened bins correspond to the probability under the null hypothesis of producing a sample with a bin height and standard deviation that is at least as extreme as the measured value. Refer to text for details.

that has been studied extensively in the mathematical literature. This link provides a rigorous mathematical basis to underpin our understanding of QBO synchronization phenomena.

Although the models derived here contain various simplifications, such as a discrete set of identical waves, a lack of density stratification, and a relatively simple wave forcing parameterization, there is no obvious reason why the results of the simple descent models would not naturally carry forward to more complex/ realistic situations. Indeed, we have seen that variations in the wave forcing profile do not engender significant qualitative changes to the structure of synchronization (cf. Figs. 5, 6, 12).

The key ingredient for the synchronization behavior in the descent models seems to be the reduction in the strength of the net wave forcing as the zero wind line approaches the tropopause. This reduction allows for significant interactions between the descending shear zone and the periodic variation in the upwelling, which is the driver of the synchronization behavior in this instance. As long as this requirement is met, which is certainly justifiable physically, the results of the simple models should carry through to more complex cases.

One drawback of our modeling approach is in the restriction of consideration to the zero wind line. In reality new QBO shear zones originate at different heights, depending on background wind conditions. Our model is not able to represent any of that variation and is restricted to generating new upper-level shear zones at the same height. The model also cannot cope with the possibility of there being no zero wind line at all in the stratosphere-or indeed, multiple zero wind lines.
The effects of the QBO-induced meridional circulation have also not been considered. As a result, some of the more complex variability of the QBO will not be reflected in the model.

We note that the wave forcing amplitudes required to drive the QBO at physically observed periods are much larger in the HL descent model as compared to the full HL partial differential equation investigated in R16. This is because our quadratic parameterization of the zonal velocity implies relatively weak shear in the descent rate model as compared to the full HL model, hence requiring stronger wave amplitudes to maintain the QBO period. In further numerical experiments (not presented here), we found that increasing the value of the diffusion coefficient in the descent model by an order of magnitude generated QBOs with both realistic periods and driving wave amplitudes.

The explicit dependence of the QBO period on diffusion in the descent rate models underscores the crucial role of diffusion in determining the period of the QBO. It is well known that the QBO generated in general circulation models is very sensitive to the choices of model setup, with the QBO period varying in response to changes in grid spacing, method of discretization, choice of dynamical core, etc. (Yao and Jablonowski 2015; Schenzinger et al. 2017). The descent rate models show why this might be the case by highlighting the influence of diffusion —or any of its proxies in a numerical model—in driving the downward propagation of the QBO.

We have shown that an analog exists between the observed seasonal onset of the QBO westerlies and the clustering of QBO periods into $n$ cycles. Furthermore, we showed that such clustering is not likely to have been 
caused by random fluctuations alone, suggesting the existence of a physical mechanism underpinning the seasonal structure of the QBO. Our study of the descent rate models has demonstrated that the clustering of QBO periods can be attributed to the modulation of the QBO descent rate by the seasonal cycle in tropical upwelling. However, this does not necessarily prove that tropical upwelling is indeed the primary driver of QBO synchronization. Other possible mechanisms should also be considered, most obviously the role of the upperstratospheric semiannual oscillation and the seasonal modulation of the wave momentum fluxes that drive the QBO descent. The latter possibility is amenable to investigation within the framework of the descent models described in this paper and will be the subject of future work.

Acknowledgments. SMO acknowledges support from the U.K. National Centre for Atmospheric Science (NCAS), as well as the U.K. Natural Environment Research Council (NERC), under the GOTHAM project (NE/P006779/1). PR acknowledges support from the U.K. Engineering and Physical Science Research Council (EPSRC), under Grant EP/K029428/1. The authors thank Lesley Gray, Peter Haynes, and Andrew Fowler for insightful discussions and feedback over the course of this work.

\section{REFERENCES}

Arnol'd, V. I., 1965: Small denominators I. Mappings of the circumference onto itself. Amer. Math Soc. Transl., Ser. 2, 46, 213-284.

Baldwin, M., and Coauthors, 2001: The quasi-biennial oscillation. Rev. Geophys., 39, 179-229, https://doi.org/10.1029/1999RG000073.

Dee, D., and Coauthors, 2011: The ERA-Interim reanalysis: Configuration and performance of the data assimilation system. Quart. J. Roy. Meteor. Soc., 137, 553-597, https://doi.org/ 10.1002/qj.828.

Dunkerton, T. J., 1990: Annual variation of deseasonalized mean flow acceleration in the equatorial lower stratosphere. J. Meteor. Soc. Japan, 68, 499-508, https://doi.org/10.2151/jmsj1965.68.4_499.

— , and D. P. Delisi, 1985: Climatology of the equatorial lower stratosphere. J. Atmos. Sci., 42, 376-396, https://doi.org/ 10.1175/1520-0469(1985)042<0376:COTELS>2.0.CO;2.

Hampson, J., and P. Haynes, 2004: Phase alignment of the tropical stratospheric QBO in the annual cycle. J. Atmos. Sci., 61, 2627-2637, https://doi.org/10.1175/JAS3276.1.
Holton, J. R., and R. S. Lindzen, 1972: An updated theory for the quasi-biennial cycle of the tropical stratosphere. J. Atmos. Sci., 29, 1076-1080, https://doi.org/10.1175/1520-0469(1972)029<1076: AUTFTQ > 2.0.CO;2.

Jensen, M. H., P. Bak, and T. Bohr, 1984: Transition to chaos by interaction of resonances in dissipative systems. I. Circle maps. Phys. Rev., 30A, 1960-1969, https://doi.org/10.1103/ PhysRevA.30.1960.

Katok, A., and B. Hasselblatt, 1997: Introduction to the Modern Theory of Dynamical Systems. Vol. 54. Cambridge University Press, 802 pp.

Kinnersley, J. S., and S. Pawson, 1996: The descent rates of the shear zones of the equatorial QBO. J. Atmos. Sci., 53, 1937-1949, https://doi.org/10.1175/1520-0469(1996)053<1937: TDROTS $>2.0$. CO;2.

Krismer, T. R., M. A. Giorgetta, and M. Esch, 2013: Seasonal aspects of the quasi-biennial oscillation in the Max Planck Institute Earth System Model and ERA-40. J. Adv. Model. Earth Syst., 5, 406-421, https://doi.org/10.1002/jame.20024.

Newman, P. A., L. Coy, S. Pawson, and L. R. Lait, 2016: The anomalous change in the QBO in 2015-2016. Geophys. Res. Lett., 43, 8791-8797, https://doi.org/10.1002/2016GL070373.

Osprey, S. M., N. Butchart, J. R. Knight, A. A. Scaife, K. Hamilton, J. A. Anstey, V. Schenzinger, and C. Zhang, 2016: An unexpected disruption of the atmospheric quasi-biennial oscillation. Science, 353, 1424-1427, https://doi.org/10.1126/science.aah4156.

Ott, E., 2002: Chaos in Dynamical Systems. Cambridge University Press, 492 pp.

Pascoe, C. L., L. J. Gray, S. A. Crooks, M. N. Juckes, and M. P. Baldwin, 2005: The quasi-biennial oscillation: Analysis using ERA-40 data. J. Geophys. Res., 110, D08105, https://doi.org/ 10.1029/2004JD004941.

Plumb, R. A., 1977: The interaction of two internal waves with the mean flow: Implications for the theory of the quasi-biennial oscillation. J. Atmos. Sci., 34, 1847-1858, https://doi.org/ 10.1175/1520-0469(1977)034<1847:TIOTIW >2.0.CO;2.

Rajendran, K., I. M. Moroz, P. L. Read, and S. M. Osprey, 2016: Synchronisation of the equatorial QBO by the annual cycle in tropical upwelling in a warming climate. Quart. J. Roy. Meteor. Soc., 142, 1111-1120, https://doi.org/10.1002/qj.2714.

Schenzinger, V., S. M. Osprey, L. Gray, and N. Butchart, 2017: Defining metrics of the quasi-biennial oscillation in global climate models. Geosci. Model Dev., 10, 2157-2016, https:// doi.org/10.5194/gmd-10-2157-2017.

Seviour, W. J. M., N. Butchart, and S. C. Hardiman, 2012: The BrewerDobson circulation inferred from ERA-Interim. Quart. J. Roy. Meteor. Soc., 138, 878-888, https://doi.org/10.1002/qj.966.

Uppala, S. M., and Coauthors, 2005: The ERA-40 Re-Analysis. Quart. J. Roy. Meteor. Soc., 131, 2961-3012, https://doi.org/ 10.1256/qj.04.176.

Yao, W., and C. Jablonowski, 2015: Idealized quasi-biennial oscillations in an ensemble of dry GCM dynamical cores. J. Atmos. Sci., 72, 2201-2226, https://doi.org/10.1175/JAS-D14-0236.1. 